\title{
Experiencia de la plastia laparoscópica de uréter retrocavo en el Hospital General de México Dr. Eduardo Liceaga
}

\author{
Experience of laparoscopic retrocave ureter plasty at Hospital General de México \\ Dr. Eduardo Liceaga
}

J. Emmanuel Rosas-Nava, Adrián A. Ramírez-Beltrán*, Luis Almazán-Treviño, Harvey M. Medrano-Urtecho, Eduardo González-Cuenca, Víctor E. Corona-Montes, Miguel Maldonado-Ávila, Juan E. Sánchez-Núñez y Jorge Jaspersen-Gastelum

Servicio de Urología, Hospital General de México Dr. Eduardo Liceaga, Ciudad de México, México

\section{Resumen}

Introducción: El uréter retrocavo es una anomalía congénita rara que requiere manejo quirúrgico. Objetivo: Reportar los resultados en el manejo laparoscópico para uréter retrocavo y hacer una revisión bibliográfica. Método: Estudio retrospectivo, descriptivo, transversal, en pacientes con diagnóstico de uréter retrocavo que fueron sometidos a plastia laparoscópica de uréter retrocavo. Resultados: Cuatro pacientes sometidos a plastia laparoscópica por diagnóstico de uréter retrocavo, 1 hombre y 3 mujeres, con una media de edad de 40.7 años. La media del tiempo operatorio fue de 138.2 minutos. La pérdida de sangre cuantificada transoperatoria media fue de $23.7 \mathrm{ml}$. Ningún paciente presentó complicaciones transoperatorias ni posoperatorias. Los pacientes permanecieron hospitalizados hasta su egreso una media de 2.5 días. Todos los pacientes presentaron mejoría de su sintomatología. Conclusiones: El uréter retrocavo es una condición rara y aún no existe un consenso sobre su diagnóstico, tratamiento y seguimiento. El manejo quirúrgico laparoscópico es una opción para el tratamiento de estos pacientes.

Palabras clave: Uréter retrocavo. Laparoscopia. Cirugía. Vena cava inferior.

\begin{abstract}
Introduction: The retrocave ureter is a rare congenital anomaly that requires surgical management. Objective: Report the results in laparoscopic management for retrocave ureter and literature review. Method: Retrospective, descriptive, crosssectional study in patients with a diagnosis of retrocave ureter who were sometimes a laparoscopic retrocave ureter. Results: Four patients sometimes underwent laparoscopic plasty for diagnosis of retrocave ureter, 1 man and 3 women, with a mean age of 40.7 years. The mean operative time was 138.2 minutes. The mean intraoperative quantified blood loss was $23.7 \mathrm{ml}$. No patient presented transoperative or postoperative complications. The patients remained hospitalized until their progress with an average of 2.5 days. All patients visualize improvement in their symptoms. Conclusion: The retrocave ureter is a rare condition and there is still no consensus on its diagnosis, treatment and follow-up. Laparoscopic surgical management is an option for the treatment of these patients.
\end{abstract}

Key words: Retrocave ureter. Laparoscopy. Surgery. Inferior vena cava.

\section{Correspondencia:}

*Adrián A. Ramírez-Beltrán

Dr. Balmis 148 


\section{Introducción}

El uréter retrocavo es una anomalía congénita rara, con una incidencia de 1 por cada 1500 nacidos vivos. La corrección quirúrgica estándar implica la extirpación del segmento retrocavo del uréter, con reubicación y reanastomosis uretero-ureteral o uretero-pélvica ${ }^{1-5}$.

El uréter retrocavo, también conocido como uréter circuncavo, es un anomalía congénita causada por un error en el desarrollo embriogénico de la vena cava inferior, resultante de la persistencia de la vena cardinal posterior caudal a la vena renal, que causa la compresión externa del uréter proximal ${ }^{1,6}$. Durante el desarrollo fetal, los segmentos prerrenal, renal y posrenal de la vena cava inferior se desarrollan a partir de las venas vitelina derecha, subcardinal derecha y sacrocardinal derecha, respectivamente, ${ }^{2,6,7}$. La embriología aberrante detrás de la vena cava preureteral es la persistencia de la vena cardinal derecha posterior, en lugar de la vena subcardinal derecha, como el segmento renal de la vena cava inferior ${ }^{8,9}$. Como la vena cardinal posterior derecha se encuentra ventral al uréter, este efectivamente se acuesta en una posición "retrocava» o "circumcava». El vaso anómalo provoca compresión del uréter derecho, llevando a diversos grados de hidronefrosis, lo que resulta en distintas presentaciones clínicas ${ }^{10-12}$.

Esta patología es más común en los hombres que en las mujeres, con una relación de 3:1', y casi exclusivamente se encuentra en el lado derecho. En general está asociada con hidronefrosis secundaria, clínicamente con presencia de dolor en el flanco derecho y malestar ${ }^{11-14}$. Los pacientes también pueden referir infecciones del tracto urinario, hematuria o fiebre.

Se han descrito dos tipos de uréter retrocavo según los hallazgos radiológicos: tipo 1 o de asa baja, tiene apariencia en forma de $S$ hasta el nivel de obstrucción, y es la variedad más prevalente $(90 \%)$, y tipo 2 o de asa alta, que comprende solo el $10 \%$ de los casos y presenta una curva del uréter derecho en forma de hoz suave, con el nivel de obstrucción en el margen lateral de la tercera vértebra lumbar'12,14,15.

La ecografía, la urografía intravenosa, la gammagrafía nuclear, la urografía por tomografía computarizada y la urografía por resonancia magnética se han utilizado en el diagnóstico de esta anomalía; de ellas, la urografía por tomografía computarizada, por su capacidad para representar la anomalía en tres dimensiones, es el método de referencia para su diagnóstico $0^{10,12,16}$.
Cuando es sintomática, la afección se trata quirúrgicamente por vía laparoscópica o cirugía abierta ${ }^{14,17}$. La uretero-ureterostomía abierta permaneció como el abordaje quirúrgico de referencia para tratar el uréter retrocavo por muchos años, pero en la última década, con el intensivo crecimiento de la cirugía mínimamente invasiva, los procedimientos laparoscópicos casi han reemplazado a la cirugía abierta debido a la recuperación más rápida asociada, el menor tiempo de estancia intrahospitalaria y los excelentes resultados cosméticos. En los primeros informes, la reparación laparoscópica del uréter retrocavo se describió con un tiempo operatorio prolongado, en gran parte debido a las dificultades iniciales con las técnicas de sutura intracorpórea ${ }^{15,18}$.

\section{Método y resultados}

Como primer paso se realizó una búsqueda avanzada en PubMed enfocada en el abordaje quirúrgico de los pacientes con uréter retrocavo, combinando los términos "Retrocaval ureter", "laparoscopy" y "surgery", que dio como resultado 62 artículos entre revisiones, estudios clínicos y reportes de caso. Los revisores trabajaron en pares, evaluaron secuencialmente los títulos, los resúmenes y luego el texto completo de todas las publicaciones identificadas, y seleccionaron 25 artículos que tenían relación con el uso de cirugía para el uréter retrocavo, de los cuales seis eran estudios de series de casos que recibieron manejo laparoscópico.

Posteriormente se realizó una revisión de los expedientes clínicos del servicio de urología del Hospital General de México Dr. Eduardo Liceaga de los últimos 5 años, para identificar aquellos pacientes con diagnóstico de uréter retrocavo sometidos a tratamiento quirúrgico laparoscópico. Se encontraron cuatro pacientes (un hombre y tres mujeres) con uréter retrocavo entre diciembre de 2015 y marzo de 2019. La edad media fue de 40.75 años (rango: 27-56 años). El índice de masa corporal tuvo una media de $24.41 \mathrm{~kg} / \mathrm{m}^{2}$ (rango: $\left.16-34.44 \mathrm{~kg} / \mathrm{m}^{2}\right)$. Dos de los cuatro pacientes tenían antecedente de oclusión tubárica bilateral y una paciente tenía antecedente de cesáreas. Dos de los cuatro pacientes presentaban antecedentes de litiasis reno-ureteral derecha. Todos los pacientes tuvieron como principal síntoma dolor lumbar derecho, dos pacientes presentaron náuseas y vómitos, y un paciente mostró síntomas del tracto urinario inferior con fiebre; ninguno de los cuatro pacientes fue diagnosticado de forma incidental. La media de tiempo desde el inicio 
de la sintomatología hasta la cirugía fue de 28 meses (rango: 4-72 meses). No se identificaron alteraciones durante la exploración física. La media de los valores de creatinina sérica fue de $0.78 \mathrm{mg} / \mathrm{dl}$ (rango: 0.6-1.1 mg/dl) y la media de la tasa de filtración glomerular estimada utilizando la ecuación de CKD-EPI 2009 fue de $104.25 \mathrm{ml} / \mathrm{min} / 1.73 \mathrm{~m}^{2}$ (rango: $75-119 \mathrm{ml} / \mathrm{min} / 1.73 \mathrm{~m}^{2}$ ). Todos los pacientes fueron evaluados con urotomografía y en dos de ellos se realizó pielografía ascendente. Los hallazgos radiológicos fueron típicos, con hidronefrosis y uréter en forma de $S$ (Fig. 1), clasificándose como tipo I en todos los pacientes según el esquema propuesto por Bateson y Atkison $^{19}$. En todos los pacientes se realizó reconstrucción laparoscópica con abordaje transperitoneal. Todas las cirugías consistieron en ureteropieloanastomosis con resección del segmento ureteral afectado (Fig. 2). En uno de los casos se realizó ureterolitotomía laparoscópica por un lito de $1 \mathrm{~cm}$ ubicado en el tercio superior del uréter derecho. El neumoperitoneo se realizó mediante técnica cerrada con aguja de Veress en la mitad de los casos y con técnica abierta con trócar de Hasson en la otra mitad. En todos los casos se insertaron tres trócares con técnica de triangulación: dos de $5 \mathrm{~mm}$ y uno de 10-12 mm paraumbilical. Tras la identificación y la disección del uréter, se cortó el uréter distal al segmento comprimido y la porción redundante de uréter proximal, así como de la pelvis renal. El uréter se colocó en posición anterior a la vena cava inferior. Con la ayuda de una guía hidrófila se insertó un catéter ureteral doble J. La anastomosis se realizó con MONOCRYL ${ }^{\circledR} 4-0$. Todos los pacientes quedaron con un drenaje tipo Penrose; la media de días hasta el retiro del drenaje fue de 3.5 (rango: 2-6 días). La media para el retiro del catéter ureteral doble $\mathrm{J}$ fue de 30 días (rango: 14-46 días). El seguimiento de los pacientes se realizó en la consulta externa del servicio de urología. Todos los procedimientos se completaron por vía laparoscópica sin necesidad de conversión a cirugía abierta. La media del tiempo operatorio fue de 138.25 minutos (rango: 102-210 minutos). La pérdida de sangre cuantificada transoperatoria media fue de $23.75 \mathrm{ml}$ (rango: $15-30 \mathrm{ml}$ ). Ningún paciente presento complicaciones transoperatorias ni posoperatorias; tampoco hubo requerimiento de transfusión sanguínea. Los pacientes permanecieron hospitalizados hasta su egreso una media de 2.5 días (rango: 2-3 días). El periodo medio de seguimiento fue de 17 meses (rango: 1-39 meses). Todos los pacientes presentaron mejoría del dolor y uno reportó resolución completa del dolor. Uno de los pacientes fue sometido a ureteroscopia derecha
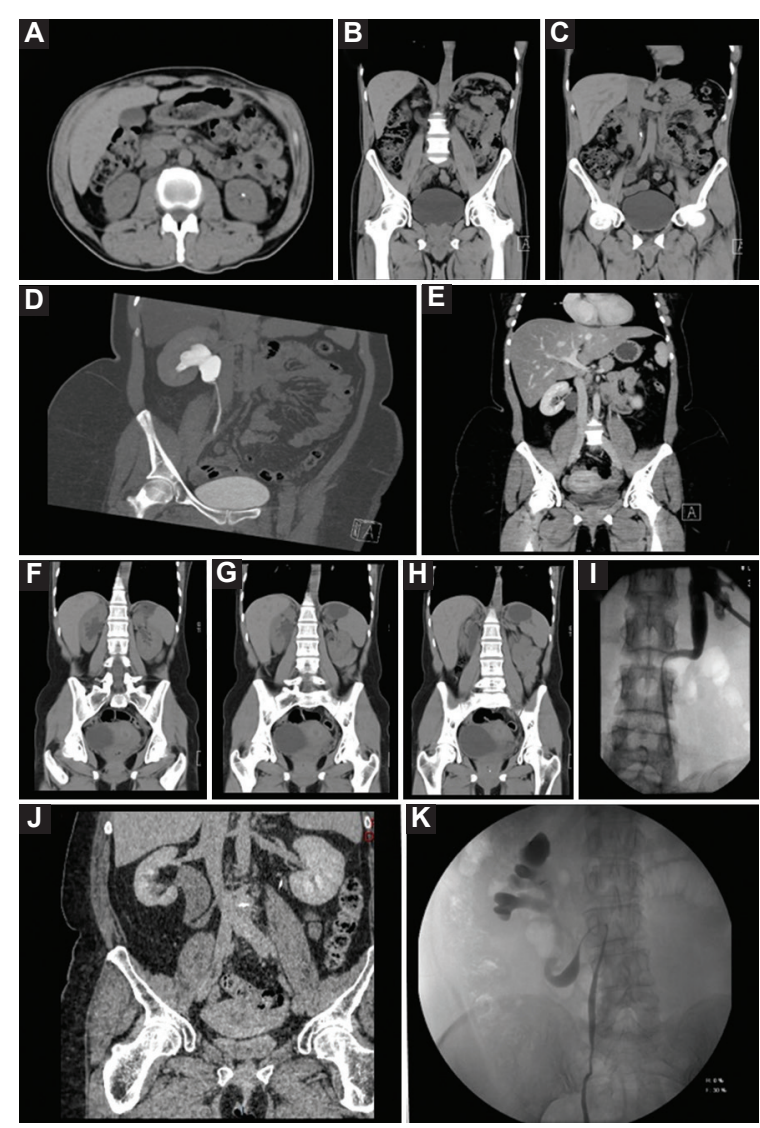

Figura 1. Hallazgos radiológicos típicos, con uréter en forma de $S$, clasificándose como tipo I en todos los pacientes según el esquema propuesto por Bateson y Atkison ${ }^{19}$. A-C: paciente 1. D y E: paciente 2. F-I: paciente 3. $\mathbf{J}$ y $\mathbf{K}$ : paciente 4.
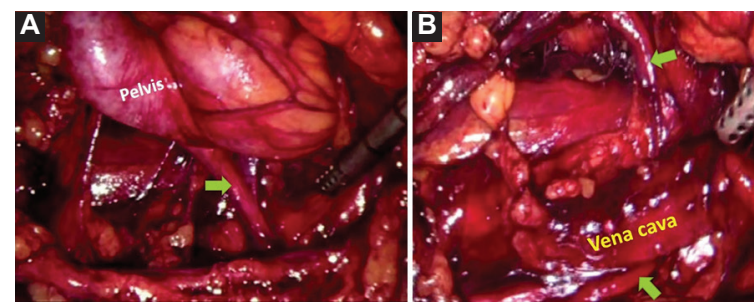

Figura 2. A: se observan la pelvis y el uréter (flecha). B: se observan la vena cava y el uréter en posición retrocava (flechas).

debido a un lito ureteral localizado en el tercio medio del uréter, con resolución completa.

\section{Discusión}

Se realizó una búsqueda en la literatura internacional y se identificaron seis estudios de series de casos de uréter retrocavo que recibieron manejo laparoscópico ${ }^{16,20-24}$. Comparamos las características clínicas y 
Tabla 1. Resultados de publicaciones sobre manejo laparoscópico de uréter retrocavo. Características clínicas y quirúrgicas

\begin{tabular}{|c|c|c|c|c|c|c|c|c|c|}
\hline Estudio & $\begin{array}{l}\text { Pacientes } \\
\text { (n) }\end{array}$ & Edad (años) & $\begin{array}{l}\text { Sexo } \\
\text { M-F }\end{array}$ & Abordaje & $\begin{array}{c}\text { Tiempo } \\
\text { quirúrgico }(\mathrm{min})\end{array}$ & $\begin{array}{c}\text { Pérdida } \\
\text { sanguínea (ml) }\end{array}$ & Complicaciones & $\begin{array}{c}\text { Días de } \\
\text { hospitalización }\end{array}$ & $\begin{array}{c}\text { Mejoría } \\
\text { sintomática } \\
(\%)\end{array}$ \\
\hline $\begin{array}{l}\text { Nuestro } \\
\text { estudio }\end{array}$ & 4 & $40.75(27-56)$ & $1-3$ & $\mathrm{TP}$ & $138.25(102-210)$ & $23.75(15-30)$ & No & $2.5(2-3)$ & 100 \\
\hline $\begin{array}{l}\text { Seo, et al. }{ }^{20} \\
(2019)\end{array}$ & 10 & $40.5(16-66)$ & $7-3$ & $\mathrm{TP}$ & $199.6(97-240)$ & $154.4(33-332)$ & No & $9.3(6-14)$ & 100 \\
\hline $\begin{array}{l}\text { Liu, et al. }{ }^{21} \\
(2016)\end{array}$ & 9 & $34.4(27-53)$ & $6-3$ & $\mathrm{RP}$ & $103(89-110)$ & ND & No & $7(6-9)$ & 100 \\
\hline $\begin{array}{l}\text { Ding, et al. }{ }^{22} \\
\text { (2012) }\end{array}$ & 9 & $35(19-47)$ & $9-0$ & $\mathrm{TP}$ & $135(70-250)$ & Mínima $(<60)$ & No & $7.3(7-10)$ & 100 \\
\hline $\begin{array}{l}\text { Chen, et al. }{ }^{23} \\
\text { (2011) }\end{array}$ & 12 & 35 (19-45) & $8-4$ & $\mathrm{RP}$ & $112(89-158)$ & $35(21-60)$ & No & $6(5-7)$ & 100 \\
\hline $\begin{array}{l}\mathrm{Li}, \text { et al. }{ }^{24} \\
(2010)\end{array}$ & 10 & $31(9-48)$ & $6-4$ & $\mathrm{RP}$ & $82(60-110)$ & Mínima $(<10)$ & No & $6.5(5-7)$ & 100 \\
\hline $\begin{array}{l}\text { Simforoosh, } \\
\text { et al. }{ }^{16}(2006)\end{array}$ & 6 & $31(16-50)$ & $3-3$ & $\mathrm{TP}$ & $180(150-210)$ & Mínimo (< 50) & No & $4(3-5)$ & 100 \\
\hline
\end{tabular}

F: femenino; M: masculino; ND: no disponible; RP: retroperitoneal; TP: transperitoneal. Los valores se presentan como medias y rangos.

quirúrgicas reportadas en estas publicaciones con los hallazgos de nuestro estudio (Tabla 1). En tres de estos estudios el abordaje fue retroperitoneal ${ }^{21,23,24}$, mientras que en los otros fue transperitoneal ${ }^{16,20,22}$; la mayor experiencia en nuestro hospital es con abordaje transperitoneal, por lo que todos nuestros pacientes fueron abordados por esta vía. La edad media que reportan los estudios es de 31 a 40.5 años, mientras que en nuestro estudio fue de 40.75 años. Con relación al sexo, nuestro estudio tuvo una relación 1:3 a favor del sexo femenino, mientras que los estudios previos refieren tener un predominio del sexo masculino. La media del tiempo quirúrgico en nuestro estudio es de 138.25 minutos, que está dentro del rango establecido de los estudios previos, que va de 82 a 199.6 minutos. La media de días de estancia hospitalaria hasta el egreso en nuestro estudio es de 2.5 días, muy inferior a la mencionados en los otros estudios, que va de 4 a 9.3 días. Otro aspecto por considerar es que, tanto en nuestro estudio como en las otras seis series de casos, ninguno de los pacientes presentó complicaciones y todos lograron una mejoría de su sintomatología.

En el año 2017 se presentó un estudio de nueve pacientes con uréter retrocavo sometidos a abordaje abierto con un tiempo quirúrgico promedio de $95.6 \pm$ 22.0 minutos (rango: 60-130 minutos), que es menor que el nuestro (media: 138.25 minutos; rango: 102-210 minutos). Reportaron un sangrado transoperatorio con una media de $106.7 \pm 32.2 \mathrm{ml}$ (rango: $50-160 \mathrm{ml}$ ), mientras que nuestra pérdida de sangre transoperatoria fue menor, con una media de $23.75 \mathrm{ml}$ (rango: 15$30 \mathrm{ml}$ ). Otro de los puntos a destacar es que el tiempo medio de hospitalización fue de $15.3 \pm 1.2$ días (rango: 14-17 días), superior al de nuestro estudio, con una media de 2.5 días de hospitalización (rango: 2-3 días) ${ }^{25}$.

Nuestros resultados son similares a los encontrados en otras series de casos manejados por vía laparoscópica; sin embargo, al compararlos con los reportados para cirugía abierta, nuestro estudio muestra que la cirugía laparoscópica ofrece la ventaja de un menor sangrado transoperatorio y un menor número de días de hospitalización, aunque el tiempo operatorio sea mayor. La primera reconstrucción laparoscópica de un uréter retrocavo fue realizada en 1994 por Baba et al. ${ }^{26}$, quienes reportaron un tiempo quirúrgico de 9.3 horas $^{20,21}$. Los avances tecnológicos y la experiencia creciente en el manejo laparoscópico han disminuido los tiempos quirúrgicos y han mejorado los resultados finales.

\section{Conclusiones}

El uréter retrocavo es una condición rara, lo que limita la información, así como la evidencia y las 
recomendaciones para su diagnóstico, tratamiento y seguimiento. Tanto la cirugía abierta como la cirugía de mínima invasión se han utilizado para tratar a los pacientes logrando una mejoría de la sintomatología. El abordaje por mínima invasión laparoscópico transperitoneal se presenta como una opción que ofrece las ventajas de un menor sangrado transoperatorio y un menor tiempo de hospitalización en comparación con la cirugía abierta. Aunque los tiempos quirúrgicos de la cirugía laparoscópica han disminuido, continúan siendo mayores que los de la cirugía abierta. Son necesarios más estudios que permitan identificar el mejor tratamiento para los pacientes.

\section{Responsabilidades éticas}

Protección de personas y animales. Los autores declaran que para esta investigación no se han realizado experimentos en seres humanos ni en animales.

Confidencialidad de los datos. Los autores declaran que han seguido los protocolos de su centro de trabajo sobre la publicación de datos de pacientes.

Derecho a la privacidad y consentimiento informado. Los autores han obtenido el consentimiento informado de los pacientes y/o sujetos referidos en el artículo. Este documento obra en poder del autor de correspondencia.

\section{Conflicto de intereses}

Los autores declaran que no existen conflictos de intereses.

\section{Bibliografía}

1. Soria GR, Reyes MA, Rodríguez FR, Lomelín JP. Uréter retrocavo: Reporte de un caso en el Hospital General de México y revisión de la literatura. Rev Med Hosp Gen Mex. 2010;73:115-9.

2. Kumar S, Shankaregowda SA, Devana SK, Jain S, Singh SK. Single-incision multiport laparoendoscopic technique to repair retrocaval ureter using the Santosh PGI ureteric tacking fixation technique. Asian J Endosc Surg. 2014;7:337-41.
3. Gundeti MS, Duffy PG, Mushtaq I. Robotic-assisted laparoscopic correction of pediatric retrocaval ureter. J Laparosc Adv Surg Tech A. 2006;16:422-4.

4. Perimenis P, Gyftopoulos K, Athanasopoulos A, Pastromas V, Barbalias G. Retrocaval ureter and associated abnormalities. Int Urol Nephrol. 2002;33:19-22.

5. Uthappa MC, Anthony D, Allen C. Case report: retrocaval ureter: MR appearances. Br J Radiol. 2002;75:177-9.

6. Junejo NN, Vallasciani S, Peters C, AlHazmi H, Almathami A, Alshammari A, et al. High retrocaval ureter: an unexpected intraoperative finding during robotic redo pyeloplasty. Urol Case Rep. 2018;20:19-21.

7. Xu DF, Yao YC, Ren JZ, Liu YS, Gao Y, Che JP, et al. Retroperitoneal laparoscopic ureteroureterostomy for retrocaval ureter: report of 7 cases. Urology. 2009;74:1242-5.

8. Chung BI, Gill IS. Laparoscopic dismembered pyeloplasty of a retrocaval ureter: case report and review of the literature. Eur Urol. 2008;54: 1433-6.

9. Fernández-Fernández JA, Pachano-Arenas FE. Laparoscopic-assisted correction of a retrocaval ureter. J Pediatr Surg. 2008;43:1560-2.

10. Mouraviev V, Polascik TJ. Laparoscopic ureteroureterostomy for retrocaval ureter. Eur Urol Suppl. 2006;5:466-9.

11. Acharya SK, Jindal B, Yadav DK, Singha S, Bagga D. Retrocaval ureter: a rare cause of hydronephrosis in children. J Pediatr Surg. 2009;44: 846-8.

12. Kajal P, Rattan $K$, Sangwan V, Bhutani N. Retrocaval ureter presenting at 6 years of age in a girl child - an extreme rarity. Asian $\mathrm{J}$ Urol. 2016;3:107-9.

13. Mejdoub I, Bouassida M, Mseddi MA, Fourati M, Hadjslimen M, Rebai N. Laparoscopic approach for retrocaval ureter: how to decrease surgical time? Urol Case Rep. 2018;20:106-7.

14. Arya MC, Kumar L, Kumar R, Mittal R. Retrocaval ureter with vesicoureteric reflux, a very rare entity. African Journal of Urology. 2017;23:5-8.

15. Gómez-Sánchez J, Sanabria-González MA, García D, López C, Correa-Rovelo JM, Sánchez-Zavala J, et al. Cirugía de mínima invasión en uréter retrocavo. Rev Invest Med Sur Mex. 2013;20:188-9.

16. Simforoosh N, Nouri-Mahdavi K, Tabibi A. Laparoscopic pyelopyelostomy for retrocaval ureter without excision of the retrocaval segment: first report of 6 cases. J Urol. 2006;175:2166-9; discussion 9.

17. Bhattacharjee S, Sanga S, Gupta P, George RA. Retrocaval ureter or preureteral vena cava: lest we forget this rare cause of hydronephrosis. Med J Armed Forces India. 2016;72(Suppl 1):S77-S9.

18. Alvarado-García R, Gallego Grijalva JE, Uribe-Ramos D, Reza-Villa A, Franco F. Uréter retrocavo en una niña de 5 años. Acta Pediatr Mex. 2002;23:65-7.

19. Bateson EM, Atkinson D. Circumcaval ureter: a new classification. Clin Radiol. 1969;20:173-7.

20. Seo IY, Oh TH, Jeon SH. Transperitoneal laparoscopic ureteroureterostomy with excision of the compressed ureter for retrocaval ureter and review of literature. Investig Clin Urol. 2019;60:108-13.

21. Liu E, Sun X, Guo H, Li F, Liu S, Wang K, et al. Retroperitoneoscopic ureteroplasty for retrocaval ureter: report of nine cases and literature review. Scand J Urol. 2016;50:319-22.

22. Ding GQ, Xu LW, Li XD, Li GH, Yu YL, Yu DM, et al. Pure transperitoneal laparoscopic correction of retrocaval ureter. Chin Med J. 2012;125: 2382-5.

23. Chen Z, Chen X, Wu ZH, Luo YC, Li NN. Treatment of retrocaval ureter by retroperitoneal laparoscopic ureteroureterostomy: experience on 12 patients. J Laparoendosc Adv Surg Tech A. 2011;21:803-7.

24. Li HZ, Ma X, Qi L, Shi TP, Wang BJ, Zhang X. Retroperitoneal laparoscopic ureteroureterostomy for retrocaval ureter: report of 10 cases and literature review. Urology. 2010;76:873-6.

25. Mao L, Xu K, Ding M, Pan J, Guo Z. Comparison of the efficacy and safety of retroperitoneal laparoscopic and open surgery for the correction of retrocaval ureter. Ther Clin Risk Manag. 2017;13:697-701.

26. Baba S, Oya M, Miyahara M, Deguchi N, Tazaki H. Laparoscopic surgical correction of circumcaval ureter. Urology. 1994;44(1):122-6. doi: 10.1016/s0090-4295(94)80023-5. 